\title{
Vasoactive-inotropic score as a predictor of in- hospital mortality in out-of-hospital cardiac arrest
}

\author{
YOUNG TAECK OH ${ }^{1}$, JAEHOON OH ${ }^{3}$, SEUNG MIN PARK ${ }^{2}$, YU JIN KIM ${ }^{2}$, YOU HWAN \\ $J^{2}$, HAE CHUL YANG ${ }^{4}$, YOUNG HWAN LEE ${ }^{5 \dagger}$, DONG KEON LEE ${ }^{2 \dagger}$ \\ 1 Department of Emergency Medicine, Armed Forces Daejeon Hospital, Republic of Korea \\ 2 Department of Emergency Medicine, Seoul National University Bundang Hospital, Republic of Korea \\ ${ }_{3}$ Department of Emergency Medicine, C ollege of Medicine, Hanyang University, Seoul, Republic of Korea \\ ${ }_{4}$ Researcher, Seoul National University Bundang Hospital, Republic of Korea \\ ${ }_{5}$ Department of Emergency Medicine, College of Medicine, Soonchunhyang University, Republic of Korea \\ $\dagger$ These authors contributed equally to this manuscript.
}

Address for Correspondence:

Dong Keon Lee

Department of Emergency Medicine, Seoul National University Bundang Hospital

13620, 82, Gumi-ro 173beon-gil, Bundang-gu, Seongnam-si, Gyeonggi-do, Republic of Korea

Phone: +82-010-8929-0908

Fax: +82-0504-428-0908

E-mail: stolenegg@gmail.com

\section{ABSTRACT}

Background. The Vasoactive-Inotropic Score (VIS) is an objective clinical tool used to quantify the need for cardiovascular support in children and adolescents after surgery and to predict prognosis of pediatric septic shock. Considering the post-cardiac arrest syndrome (PCAS) is a sepsis-like syndrome, we aimed to investigate the correlation between VIS and inhospital mortality in out-of-hospital cardiac arrest (OHCA) patients who achieved a sustained return of spontaneous circulation (ROSC) and admitted to the intensive care unit (ICU).

Methods. A retrospective chart review of 504 OHCA patients who were admitted to the emergency room with OHCA from Jan 2015 to Dec 2016 was done. VIS was calculated with the recorded administration rate of the drugs on electronic medical record at the same time during the first 24 hours in ICU. The highest value of VIS in 24 hours (24hr-peak VIS) was used for investigating the correlation between VIS and in-hospital mortality.

Results. Among 504 OHCA patients, 166 patients were admitted to the intensive care unit and 116 patients died during hospital stay. The probability of in-hospital mortality was significantly higher when 24hr-peak VIS was higher than 33.3 [Odds ratio $(\mathrm{OR})=3.18,95 \% \mathrm{CI}=1.22-8.29, \mathrm{p}$ value $=0.018]$.

Conclusion. 24hr-Peak VIS could be a good scoring system for predicting in-hospital mortality in OHCA patients who admitted to ICU. The AUC was 0.762 (95\% CI
$=0.690$ to 0.825$)$ and the optimal cut-off values were 33.3 (sensitivity 0.764 , specificity 0.610).

\section{BACKGROUND}

Sudden cardiac death (SCD), unexpected death from a cardiovascular cause that occurs outside of the hospital, remains a significant public health problem estimated to account for 15 - $20 \%$ of all deaths(1)]. Moreover, the survival to hospital discharge rate after out-of-hospital cardiac arrest (OHCA) has remained poor over 30 years despite the major advances in cardiopulmonary resuscitation (CPR) practice(2).

In OHCA patients, early prognostication is important in the aspects of medical ethics as well as planning treatment plans. Although there have been many efforts for predicting prognosis of OHCA patients, no single standard predictor was developed(3).

The Vasoactive-Inotropic Score (VIS) is an objective clinical tool used to quantify the need for cardiovascular support in children and adolescent after surgery(4-7) and to predict prognosis of pediatric septic $\operatorname{shock}(8)$.

Given that the post-cardiac arrest syndrome (PCAS) is a sepsis-like syndrome(9), and VIS can predict the prognosis of septic shock, we hypothesised that VIS could predict the prognosis of post-cardiac arrest patients. To the authors' knowledge, there was no study about the correlation of VIS and outcome of OHCA. Therefore, we con- ducted this study to investigate the association between VIS and OHCA outcomes.

\section{METHODS}

\section{Study design and data collection}

This was a retrospective and observational study. Consecutive Adult OHCA patients who achieved ROSC and were admitted to the hospital from Jan 2015 to Dec 2016, were included. Exclusion criteria were age $<18$ years, traumatic cardiac arrest, history of terminal malignancy, cerebral performance category $(\mathrm{CPC}) \geq 3$ score prior to cardiac arrest.

Age, gender, the location of arrest, witnessed cardiac arrest, initial rhythm, and bystander CPR, the cause of cardiac arrest, $\mathrm{CPR}$ duration and targeted temperature management, the rate of survival to admission and neurologic outcome at discharge were collected from the emergency department cardiac arrest registry.

For calculating VIS, administration rate of vasoactive drugs and inotropes was reviewed from the electronic medical record (EMR). Two trained researchers reviewed the registry and EMR. If there was a conflict, one emergency medicine specialist reviewed that case.

\section{Vasoactive-inotropic score (VIS)}

Vasoactive drugs and inotropes were administered to maintain the mean arterial pressure above $65 \mathrm{mmHg}$. The vital signs 
and administration rate of vasoactive drugs and inotropes were recorded every hour unless there were unstable events. VIS was calculated with the recorded administration rate of the drugs at the same time during the first 24 hours in ICU using the following formulae(10-11):

At least 24 values of VIS were obtained per each patient and the highest value of VIS within 24 hours (24hr-peak VIS) after admission to the ICU was used for further analysis.

\section{Primary and secondary outcomes}

The primary outcome of this study was the probability of in-hospital mortality correlated with VIS. The secondary outcome was the probability of neurologically intact survival correlated with VIS. Neurologically intact survival (NIS) group was defined as the patients who were discharged alive with CPC score of 1-2(12) and nonNIS group was defined as the patients who died in hospital or were discharged alive with CPC score of 3-5.

\section{Statistical analysis}

Statistical analyses were performed using SPSS software for Windows (V.20.0 K, SPSS, Chicago, IL, USA). Nominal data are presented as frequencies and percentages, and continuous variables are presented as the mean and standard deviation (SD) and median and interquartile range (IQR) after assessments for normality using the Shapiro-Wilk test. The chi-square test or Fisher's exact test were used for comparisons of nominal variables, while the two-sample ttest and Mann-Whitney U-test were used to compare continuous variables. Predictors of poor prognosis were determined by logistic regression analysis, and optimal cut-off points of predictors were evaluated by receiver operation characteristic (ROC) curves and the Youden index. P-values less than 0.05 were considered statistically significant.

Multivariate logistic regression analysis was used to identify independent predictors of in-hospital mortality and neurologically intact survival at hospital discharge, as measured by the estimated odds ratio (OR) with 95\% confidence intervals (CIs). Variables with a p-value less than 0.2 on univariate analyses as well as clinically relevant variables were entered into the forward stepwise multiple logistic regression models.

\section{Power analysis}

Statistical power was calculated using $\mathrm{G}^{\star}$ Power 3.1, and the primary outcome was the probability of in-hospital mortality correlated with VIS. Given the probabilities of in-hospital mortality were 0.779 , 0.410 in the patients whose $24 \mathrm{hr}$-peak VIS was 33.3 or higher and lower than 33.3 respectively, and sample size was 166 , the type 1 error $<0.001$ and power $>0.999$ were calculated for a two-tail logistic regression.

\section{RESULTS}

504 OHCA patients were admitted to the emergency room (ER) during the study period. Among them, 285 patients were excluded due to age $(\mathrm{n}=11)$, traumatic cardiac arrest $(n=57)$ and death at $E R(n=217)$. A total of 219 patients were admitted to

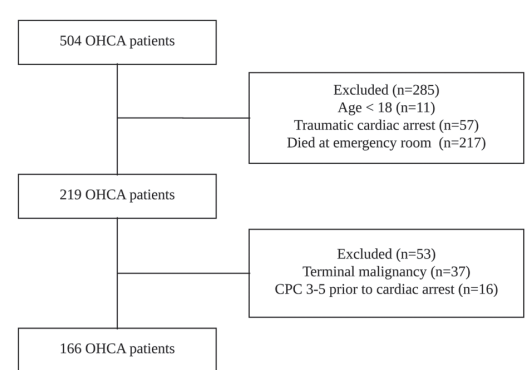

Figure 1. Flow diagram

OHCA: Out-of-hospital cardiac arrest, CPC: Cerebral performance category

the ICU, and 53 patients were excluded because of terminal malignancy $(n=37)$ and CPC 3-5 prior to cardiac arrest $(\mathrm{n}=16)$ (Fig. 1).

As a result, 166 patients were included in this study. Among them, 60 patients survived at discharge, and 36 patients showed a neurologically intact survival. Fortyseven $(73.4 \%)$ versus 55 patients $(51.8 \%)$ were men and the mean age was $56.6 \pm$ 18.3 versus $62.4 \pm 16.4$ in survivors and non-survivors respectively ( $\mathrm{p}<0.001$ and $\mathrm{p}=0.015$, respectively). Also, there were significant differences between survivors and non-survivors regarding the cause of cardiac arrest, the location of cardiac arrest, initial shockable rhythm and CPR duration (Table 1 ).

Twenty-nine (80.6\%) versus 73 patients (56.2\%) were men, and the mean age was $47.8 \pm 13.7$ versus $63.3 \pm 16.8$ in the NIS group and the non-NIS group respectively ( $\mathrm{p}=0.011$ and $\mathrm{p}<0.001$, respectively). The NIS group showed a higher rate of cardiac cause, public area, initial shockable rhythm

Table 1. Clinical characteristics ${ }^{*}$

\begin{tabular}{lllllll}
\hline & $\begin{array}{l}\text { Survivors } \\
(\mathbf{n}=\mathbf{6 0})\end{array}$ & $\begin{array}{l}\text { Non-survivors } \\
(\mathbf{n}=\mathbf{1 0 6})\end{array}$ & $\mathbf{p}$ & $\begin{array}{l}\text { NIS group } \\
(\mathbf{n = 3 6})\end{array}$ & $\begin{array}{l}\text { Non-NIS group } \\
(\mathbf{n}=\mathbf{1 3 0})\end{array}$ & $\mathbf{P}$ \\
\hline Age (years) & $55.6 \pm 18.3$ & $62.4 \pm 16.4$ & $0.015^{*}$ & $47.8 \pm 13.7$ & $63.3 \pm 16.8$ & $<0.001^{*}$ \\
\hline Male & $47(78.3)$ & $55(51.9)$ & $0.001^{*}$ & $29(80.6)$ & $73(56.2)$ & $0.011^{*}$ \\
\hline Cardiac cause & $34(56.7)$ & $25(23.6)$ & $<0.001^{*}$ & $28(77.8)$ & $31(23.8)$ & $<0.001^{\star}$ \\
\hline Public area & $30(50.0)$ & $36(34.0)$ & $0.047^{*}$ & $22(61.1)$ & $44(33.8)$ & $0.003^{*}$ \\
\hline Witnessed arrest & $37(61.7)$ & $70(66.0)$ & 0.572 & $22(61.1)$ & $85(65.4)$ & 0.635 \\
\hline Bystander CPR & $32(53.3)$ & $50(47.2)$ & 0.445 & $23(63.9)$ & $59(45.4)$ & 0.06 \\
\hline Shockable rhythm & $27(45.0)$ & $16(15.1)$ & $<0.001^{*}$ & $24(66.7)$ & $19(14.6)$ & $<0.001^{*}$ \\
\hline 24hr-peak VIS & $29.2(0-83.9)$ & $97.6(30.6-196.8)$ & $<0.001^{*}$ & $17.0(0-66.5)$ & $88.2(28.6-183.0)<0.001^{*}$ \\
\hline CPR duration & $27 \pm 12$ & $32 \pm 13$ & $0.019^{*}$ & $20 \pm 9$ & $32 \pm 13$ & $<0.001^{*}$ \\
\hline TTM & $19(41.3)$ & $27(58.7)$ & 0.471 & $32(24.6)$ & $14(38.9)$ & 0.097
\end{tabular}

Data are expressed as mean \pm SD, number (\%), or median (IQR), 24hr-peak VIS: The highest value of vasoactive-inotropic score in 24 hours, TTM: Target Temperature Management, NIS: Neurologically intact survival

${ }^{*} \mathrm{p}$ value $<0.05$ 
Table 2. Multivariate logistic regression analysis

\begin{tabular}{lll}
\hline & \multicolumn{2}{l}{ Neurologically intact survival at hospital discharge } \\
\hline Male & OR $(\mathbf{9 5} \% \mathbf{C I})$ & $\mathbf{P}$ \\
\hline Age $\geq 65$ & $1.91(0.43-8.57)$ & 0.397 \\
\hline Public area & $16.22(2.73-96.43)$ & $0.002^{*}$ \\
\hline Witnessed arrest & $0.86(0.24-3.10)$ & 0.819 \\
\hline Bystander CPR & $0.94(0.28-3.20)$ & 0.923 \\
\hline Shockable rhythm & $0.36(0.09-1.44)$ & 0.147 \\
\hline Cardiac cause & $0.08(0.02-0.32)$ & $<0.001^{*}$ \\
\hline CPR duration & $1.40(0.38-5.23)$ & 0.613 \\
\hline 24hr-peak VIS $>33.3$ & $0.97(0.94-1.01)$ & 0.121 \\
\hline TTM & $1.18(0.31-4.55)$ & 0.811 \\
\hline
\end{tabular}

24hr-peak VIS: The highest value of vasoactive-inotropic score in 24 hours, TTM: Target Temperature Management

${ }^{*} \mathrm{p}$ value $<0.05$

and shorter duration of CPR (Table 1). Regarding VIS, the 24hr-peak VIS was significantly lower in survivors compared to non-survivors [29.2 (IQR 0-83.9) versus 97.6 (IQR 30.6-196.8), $\mathrm{p}<0.001$ ]. Also, the NIS group showed lower 24hr-peak VIS compared to the non-NIS group [17.0 (IQR 0 - 66.5) versus 88.2(IQR 28.6 183.0), $\mathrm{p}<0.001$ ] (Table 1).

The ROC curve analysis was performed for the prediction of in-hospital mortality and neurologically intact survival. The AUCs of the 24hr-peak VIS for the prediction of in-hospital mortality were $0.762(95 \% \mathrm{CI}=$ 0.690 to 0.825 ) and the optimal cut-off values were 33.3 (sensitivity 0.764 , specificity 0.610) (Fig. 2). Regarding the neurologically intact survival, the AUC of $24 \mathrm{hr}$-peak VIS was $0.763(95 \% \mathrm{CI}=0.691$ to 0.826$)$ with the optimal cut-off value of 33.3 (sensitivity 0.694 , specificity 0.721 ).

In the univariate logistic regression model, the probability of in-hospital mortality increases as 24hr-peak VIS increases [Odds ratio $(\mathrm{OR})=1.0107,95 \% \mathrm{CI}=1.0056$ - 1.0159, p < 0.001] (Fig. 3). In the multivariate logistic regression model, the probability of in-hospital mortality was significantly higher when $24 \mathrm{hr}$-peak VIS was higher than 33.3 [Odds ratio $(\mathrm{OR})=$ $3.18,95 \% \mathrm{CI}=1.22-8.28$, $\mathrm{p}$ value $=0.018]$ (Fig. 4). However, there was no significant correlation between 24hr-peak VIS and the neurologically intact survival (OR $=1.18,95 \% \mathrm{CI}=0.26-4.17)$, $\mathrm{p}$ value $=$ 0.951) (Table 2).

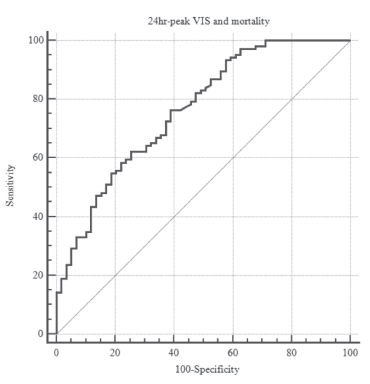

Figure 2a. ROC curve analysis for the prediction of in-hospital mortality

ROC: receiver operation characteristic, 24hr-peak VIS: the highest value of vasoactive-inotropic score in 24 hours, ${ }^{*} p$ value $<0.05$

24hr-peak VIS showed an AUC of 0.762 (95\% CI $=0.690$ to 0.825 ) and the optimal cut-off values were 33.3 (sensitivity 0.764 , specificity 0.610).

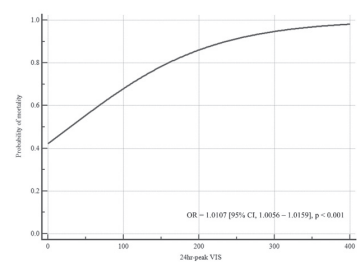

Figure 3. Univariate logistic regression for in-hospital mortality of OHCA patients

24hr-peak VIS: The highest value of vasoactive-inotropic score in 24 hours

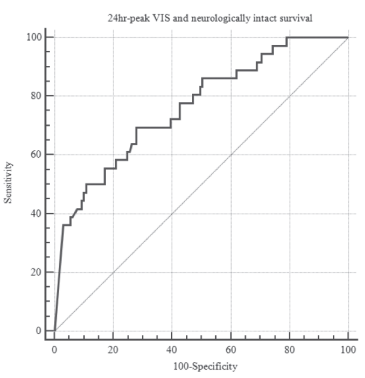

Figure 2b. ROC curve analysis for the prediction of neurologically intact survival at hospital discharge

ROC: receiver operation characteristic, 24hr-peak VIS: the highest value of vasoactive-inotropic score in 24 hours, ${ }^{*} \mathrm{p}$ value $<0.05$

24hr-peak VIS showed an AUC of 0.763 (95\% CI $=0.691$ to 0.826$)$ with the optimal cut-off value of 33.3 (sensitivity 0.694 , specificity 0.721 ).

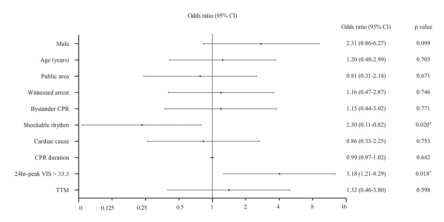

Figure 4. Multivariate logistic regression for in-hospital mortality of OHCA patients

24hr-peak VIS: The highest value of vasoactive-inotropic score in 24 hours, TTM: Target Temperature Management, ${ }^{*} \mathrm{p}$ value $<0.05$ 


\section{DISCUSSION}

In the present study, we sought to investigate the association between VIS and outcomes of OHCA patients. As a result, we reported that the value of the $24 \mathrm{hr}$-peak VIS higher than 33.3 showed a higher probability of in-hospital mortality.

Many efforts were made for predicting prognosis in OHCA patients which is essential for the treatment plan and ethics. However, no single method could predict the outcome. Moreover, only a few methods could be used in the early phase of the post-resuscitation period.

In the prehospital phase, the presence of the initial shockable rhythm and bystander CPR could predict the better outcome (13) After ROSC (return of spontaneous circulation), status myoclonus within 24 hours predicts unfavourable neurological outcome in comatose patients with OHCA treated with therapeutic hypothermia(14). Regarding blood markers, serum ammonia and lactate levels on arrival are used as the prognostic factors(15), and higher copeptin at admission, DNI (delta neutrophil index), serial NSE (neuron-specific enolase) and S-100B protein were reported as being associated with poor outcome(16-19). In terms of image study, grey matter to white matter ratio (GWR) and optic nerve sheath diameter (ONSD) on initial brain CT cor- related with the neurologic outcome of hypoxic-ischemic encephalopathy. $(3,20)$ However, these blood markers and image studies could not be measured continuously or immediately.

There have been many studies for predicting the outcome using scoring systems such as SAPS III, OHCA score, RACA score(21-24). However, SAPS III could not predict mortality(23), and RACA score could predict only ROSC but not the Neurologically intact survival nor in-hospital mortality (25). OHCA score, one of the predictors of OHCA, has the limitation as it contains no-flow interval and low-flow interval which are difficult to be estimated or recorded in a large number of OHCA patients(1224) Recently, Argaud et al. reported that SOFA score at ICU admission was independently associated with 28-day mortality(26).

VIS is a clinical scoring system used to predict prognosis in the patients had cardiac surgery and with sepsis $(4-8,10,11,27)$. As PCAS is one of the sepsis-like syndromes and has the component of cardiac dysfunction, we investigated the correlation between VIS and in-hospital mortality of the post-resuscitated patients.

There are some limitations of the present study. First, this study was investigated in a single tertiary university hospital. Since our hospital had a protocol which was made to maintain the mean arterial pressure above $65 \mathrm{mmHg}$, other hospitals that have a higher blood pressure target protocol, may not be able to use the same cut-off value.

Second, though the NIS group showed lower 24hr-peak VIS than the non-NIS group, the long-term neurologic outcome should be investigated to make a strong conclussion.

\section{CONCLUSION}

24hr-Peak VIS could be used to predict inhospital mortality in OHCA patients. The AUC was 0.762 (95\% CI $=0.690$ to 0.825$)$ and the cut-off value was 33.3 .

\section{Conflicts of interest}

None

\section{ACKNOWLEDGEMENTS}

This work was supported by the Soonchunhyang University Research Fund.

The authors thank Division of Statistics in Medical Research Collaborating Center at Seoul National University Bundang Hospital for statistical analyses.

\section{REFERENCES}

1. Hayashi M, Shimizu W, Albert CM. The spectrum of epidemiology underlying sudden cardiac death. Circ Res 2015;116:1887-906. doi:10.1161/CIRCRESAHA.116.304521.

2. Yang HJ, Kim GW, Kim H, Cho JS, Rho TH, Yoon HD et al. Epidemiology and outcomes in out-of-hospital cardiac arrest: a report from the NEDIS-based cardiac arrest registry in Korea. J Korean Med Sci 2015;30:95-103. doi:10.3346/jkms.2015.30.1.95.

3. Lee YH, Oh YT, Ahn HC, Kim HS, Han SJ, Lee JJ et al. The prognostic value of the grey-to-white matter ratio in cardiac arrest patients treated with extracorporeal membrane oxygenation. Resuscitation 2016;99:50-5. doi:10.1016/j.resuscitation.2015.11.009.

4. Butts RJ, Scheurer MA, Atz AM, Zyblewski SC, Hulsey TC, Bradley SM et al. Comparison of maximum vasoactive inotropic score and low cardiac output syndrome as markers of early postoperative outcomes after neonatal cardiac surgery. Pediatr Cardiol 2012;33:6338

5. Davidson J, Tong S, Hancock H, Hauck A, Da Cruz E, Kaufman J. Prospective validation of the vasoactive-inotropic score and correlation to short-term outcomes in neonates and infants after cardiothoracic surgery. Intensive Care Med 2012;38:1184-90

6. Garcia RU, Walters III HL, Delius RE, Aggarwal S. Vasoactive inotropic score (VIS) as biomarker of short-term outcomes in adolescents after cardiothoracic surgery. Pediatr Cardiol2016;37:271-7

7. Kumar M, Sharma R, Sethi SK, Bazaz S, Sharma P, Bhan A et al. Vasoactive Inotrope Score as a tool for clinical care in children post cardiac surgery. Indian J Crit Care Med 2014;18:653

8. Haque A, Siddiqui N, Munir O, Saleem S, Mian A. Association between vasoactive-inotropic score and mortality in pediatric septic shock. Indian Pediatr.2015;52:311-3

9. Adrie C, Adib-Conquy M, Laurent I, Monchi M, Vinsonneau C, Fitting C et al. Successful cardiopulmonary resuscitation after cardiac arrest as a "sepsis-like" syndrome. Circulation 2002;106:562-8

10. Gaies MG, Gurney JG, Yen AH, Napoli ML, Gajarski RJ, Ohye RG et al. Vasoactive-inotropic score as a predictor of morbidity and mortality in infants after cardiopulmonary bypass. Pediatr Crit Care Med2010;11:234-8

11. Sanil Y, Aggarwal S. Vasoactive-inotropic score after pediatric heart transplant: A marker of adverse outcome. Pediatr Transplant 2013;17:567-72

12. Jacobs I, Nadkarni V, Bahr J, Berg RA, Billi JE, Bossaert L et al. Cardiac arrest and cardiopulmonary resuscitation outcome reports: update and simplification of the Utstein templates for resuscitation registries.: A statement for healthcare professionals from a task force of the international liaison committee on resuscitation (American Heart Association, European Resuscitation Council, Aus- 
tralian Resuscitation Council, New Zealand Resuscitation Council, Heart and Stroke Foundation of Canada, InterAmerican Heart Foundation, Resuscitation Council of Southern Africa). Resuscitation2004;63:233-49

13.Zhang Q, Qi Z, Liu B, Li C. Predictors of survival and favorable neurological outcome in patients treated with targeted temperature management after cardiac arrest: A systematic review and meta-analysis. Heart Lung2018;47(6):602- 9. doi:10.1016/j. hrtlng.2018.07.005.

14. Ruknuddeen MI, Ramadoss R, Rajajee V, Grzeskowiak LE, Rajagopalan RE. Early clinical prediction of neurological outcome following out of hospital cardiac arrest managed with therapeutic hypothermia. Indian J Crit Care 2015;19:304-10. doi:10.4103/09725229.158256.

15. Shinozaki K, Oda S, Sadahiro T, Nakamura M, Hirayama Y, Watanabe E et al. Blood ammonia and lactate levels on hospital arrival as a predictive biomarker in patients with out-of-hospital cardiac arrest. Resuscitation 2011;82:404-9. doi:https://doi.org/10.1016/j. resuscitation.2010.10.026.

16. Ristagno G, Latini R, Plebani M, Zaninotto M, Vaahersalo J, Masson S et al. Copeptin levels are associated with organ dysfunction and death in the intensive care unit after out-of-hospital cardiac arrest. Crit Care 2015;19:132. doi:10.1186/s13054-015-0831-y.

17. Yune HY, Chung SP, Park YS, Chung HS, Lee HS, Lee JW et al. Delta neutrophil index as a promising prognostic marker in out of hospital cardiac arrest. PloS one. 2015;10:e0120677

18. Stammet P, Collignon O, Hassager C, Wise MP, Hovdenes J, Åneman A et al. Neuron-specific enolase as a predictor of death or poor neurological outcome after out-of-hospital cardiac arrest and targeted temperature management at $33 \mathrm{C}$ and $36 \mathrm{C}$. J Am Coll Cardiol2015;65:2104-14

19. Choi S, Park K, Ryu S, Kang T, Kim H, Cho S et al. Use of S-100B, NSE, CRP and ESR to predict neurological outcomes in patients with return of spontaneous circulation and treated with hypothermia. Emerg Med J 2016;emermed-2015-205423

20. Hwan Kim Y, Ho Lee J, Kun Hong C, Won Cho K, Hoon Yeo J, Ju Kang M et al. Feasibility of optic nerve sheath diameter measured on initial brain computed tomography as an early neurologic outcome predictor after cardiac arrest. Acad Emerg Med2014;21:11218. doi:10.1111/acem.12477.

21. Grasner JT, Meybohm P, Lefering R, Wnent J, Bahr J, Messelken M et al. ROSC after cardiac arrest--the RACA score to predict outcome after out-of-hospital cardiac arrest. Eur Heart J 2011;32:1649-56. doi:10.1093/eurheartj/ehr107.

22. Rittenberger JC, Tisherman SA, Holm MB, Guyette FX, Callaway CW. An early, novel illness severity score to predict outcome after cardiac arrest. Resuscitation 2011;82:1399-404. doi:10.1016/j.resuscitation.2011.06.024.

23. Bisbal M, Jouve E, Papazian L, de Bourmont S, Perrin G, Eon B et al. Effectiveness of SAPS III to predict hospital mortality for postcardiac arrest patients. Resuscitation 2014;85:939-44

24. Adrie C, Cariou A, Mourvillier B, Laurent I, Dabbane H, Hantala F et al. Predicting survival with good neurological recovery at hospital admission after successful resuscitation of out-of-hospital cardiac arrest: the OHCA score. Eur Heart J 2006;27:2840-5

25. Gräsner J-T, Meybohm P, Lefering R, Wnent J, Bahr J, Messelken M et al. ROSC after cardiac arrest-the RACA score to predict outcome after out-of-hospital cardiac arrest. Eur Heart J.2011;32:1649-56

26. Cour M, Bresson D, Hernu R, Argaud L. SOFA score to assess the severity of the post-cardiac arrest syndrome. Resuscitation 2016;102:110-5

27. McIntosh A, Tong S, Deakyne S, Davidson J, Scott H. Validation of the Vasoactive-Inotropic Score in Pediatric Sepsis. Pediatr Crit Care Med. 2017 Aug;18(8):750-757. doi: 10.1097/PCC.0000000000001191. 